\title{
Tangence
}

\section{Guerre, lettres et devenir historique de la Nouvelle-France dans La défaite des Sauvages armouchiquois de Marc Lescarbot}

\section{War, letters and the progress of history in New France in Marc Lescarbot's La défaite des Sauvages armouchiquois}

\author{
Isabelle Lachance
}

Numéro 111, 2016

Guerre et texte sous l'Ancien Régime : réécriture, procédés et enjeux

URI : https://id.erudit.org/iderudit/1038510ar

DOI : https://doi.org/10.7202/1038510ar

Aller au sommaire du numéro

Éditeur(s)

Tangence

ISSN

1189-4563 (imprimé)

1710-0305 (numérique)

Découvrir la revue

Citer cet article

Lachance, I. (2016). Guerre, lettres et devenir historique de la Nouvelle-France dans La défaite des Sauvages armouchiquois de Marc Lescarbot. Tangence,

(111), 131-142. https://doi.org/10.7202/1038510ar
Résumé de l'article

Dans son poème épique La défaite des Sauvages armouchiquois par le sagamos Membertou et ses alliez sauvages de 1607, Marc Lescarbot érige Pierre Dugua de Mons, vice-roi d'Henri IV en Acadie, et Jean de Biencourt de Poutrincourt, vice-lieutenant de Dugua de Mons, en pacificateurs de tribus amérindiennes irrépressiblement guerrières. Dès lors, bien que les genres de l'histoire universelle et de l'épopée semblent agir en tant que dispositifs de reconnaissance pour signifier le partage d'une commune humanité entre Souriquois, alliés locaux, et Français, porteurs de l'entreprise coloniale, une lecture parallèle de La défaite et de certains chapitres de l'Histoire de la Nouvelle France de 1609, soit « Des Lettres » et " De la Guerre », montre plutôt une mise à l'écart symbolique de l'Amérindien. Non seulement parce que cette geste quasi médiévale relègue son expérience du territoire à un temps révolu, voire à la fable - laissant ainsi le champ libre à une expérience européenne appartenant d'emblée au temps présent -, mais aussi parce qu'elle déclare l'incapacité du Sauvage à faire face au temps présent. Ainsi se voit-il non seulement doublement dominé par la différence de l'écriture en tant qu'objet du récit et par la maîtrise de l'écriture qui donne à l'Européen un ascendant conférant à sa prise de pouvoir un caractère naturel, mais encore La défaite représente les Souriquois remportant la bataille spécifiquement parce qu'ils auraient déployé à l'encontre de leurs ennemis non seulement leurs propres ressources - stratégiques et matérielles -, mais bien le tonnerre des armes à feu françaises. 
Tangence $\square \mathrm{n}^{\circ} 111,2016$, p. 131-142.

\section{Guerre, lettres et devenir historique de la Nouvelle-France dans La défaite des Sauvages armouchiquois de Marc Lescarbot \\ Isabelle Lachance \\ Université du Québec à Trois-Rivières}

L'Histoire de la Nouvelle France de Marc Lescarbot, publiée en 1609 puis augmentée jusqu'en 1618, de même que ses Muses de la Nouvelle France, recueil poétique accompagnant chaque édition de l'Histoire, sont les titres qui viennent spontanément à l'esprit quand il est question de l'histoire coloniale sous Henri iv. Mais le premier écrit substantiel publié par Lescarbot à son retour en France, à la suite d'un séjour d'un an à Port-Royal, est bien La défaite des Sauvages armouchiquois par le sagamos Membertou et ses alliez sauvages de 1607 . Ce poème épique de quelque 500 alexandrins ${ }^{1}$ relate un épisode guerrier opposant les Souriquois, Micmacs alliés des Français en Acadie, aux Armouchiquois, peuple «aux meilleures terres de toute la coste d'Arcadie [sic]», si l'on en croit Des sauvages de Champlain (1603), récit qui, en les qualifiant de «monstrueux ${ }^{2} »$, les désignait déjà comme ennemis - à la fois des alliés des Français

1. On prendra connaissance de la réception de La défaite dans l' «Avant-texte» de Marie-Christine Pioffet à: Marc Lescarbot, Poésies et opuscules sur la NouvelleFrance, éd. Marie-Christine Pioffet et Isabelle Lachance, Montréal, Nota bene, coll. «Galliana», 2014, p. 20 et suiv.

2. «[C]ar leur teste est petite, explique-t-il, et le corps court, les bras menus comme d'un schelet, et les cuisses semblablement: les jambes grosses et longues, qui sont toutes d'une venue, et quand ils sont assis sur leurs talons, les genoux leur passent plus d'un demy pied par-dessus la teste, qui est chose estrange, et semblent estre hors de nature» (Samuel Champlain, Des Sauvages, Paris, Claude de Monstr'œil, 1603, fo xxxiv $\mathrm{r}^{\mathrm{o}}$ ). 
et de l'humanité même. Entreprise par les Souriquois, la bataille relatée dans La défaite avait pour objectif de venger le meurtre de leur chef, Panoniac. Dans l'objectif de cerner de quelle manière l'imaginaire de la guerre dans l'Ancien Régime contribue à la promotion coloniale chez Lescarbot - et, par conséquent, comment il s'intègre à son travail littéraire en appui à la Nouvelle-France -, ce poème sera lu ici au prisme des chapitres «Des Lettres» et «De la Guerre» du troisième et dernier livre de l'édition de 1609 de son Histoire.

\section{La défaite dans la production de Lescarbot}

Selon Éric Thierry, le canevas narratif de La défaite découlerait des entretiens qu'aurait eus Lescarbot avec Semcoudech, Souriquois «qui faisait le voyage de France et qui avait participé à [cette] expédition $^{3}{ }$. Bien que sa forme rimée l'éloigne de la simple chronique, ce récit vient répondre à un intérêt grandissant pour l'actualité, comme le feront l'Histoire de Lescarbot en 1609 et ses publications ponctuelles sur l'évolution de la Nouvelle-France que seront $\mathrm{La}$ conversion des Sauvages de 1610 et la Relation dernière de ce qui s'est passé au voyage du sieur de Poutrincourt de 1612. La défaite vient en outre ajouter à une histoire militaire déjà présente au catalogue de l'imprimeur Jérémie Périer, qui présentait déjà des titres relatant le siège d'Ostende ou encore la bataille de Gibraltar, récapitulée dans un Discours de la rencontre des deux armées navales, d'Espagne et de Hollande [...] au destroit de Gibraltar ${ }^{4}$. Plus particulièrement, le texte de Lesarbot s'inscrit dans le genre des récits de défaites, courants à la fin du XVI ${ }^{\mathrm{e}}$ et au début du XVII ${ }^{\mathrm{e}}$ siècles: défaites de huguenots à La Rochelle ou à Albi, défaites d'infidèles aux mains des chrétiens, défaites des Maures et des Turcs aux mains des Espagnols et des Perses ${ }^{5}$.

3. Éric Thierry, La France de Henri iv en Amérique du Nord. De la création de l'Acadie à la fondation de Québec, Paris, Honoré Champion, coll. «Les géographies du monde», 2008, p. 272.

4. Il s'agit de l'Histoire remarquable et veritable de ce qui s'est passé par chacun jour au siege de la ville d'Ostende, de part et d'autre jusques à present, Paris, Jérémie Perier, 1604 et du Discours de la rencontre des deux armées navales, d'Espagne et de Hollande et des succès de leur combat faict au destroit de Gibraltar le 7 de may 1607, Paris, Jérémie Perier, 1604.

5. On en prendra pour exemples le Récit du succez et journée que le grand commandeur de Castille a eu, allant avec vingt-cinq galères contre les Mores, qui se sont nouvellement eslevés, en la montagne de Frexiliana près Malaga et de la défaite d'iceux [...], Lyon, B. Rigaud, 1569; la Defaitte des Reistres et autres rebelles, par 
Dans la chronologie des publications de Lescarbot promouvant la jeune colonie acadienne, seul un Adieu à la France ${ }^{6}$, poème d'une centaine d'alexandrins publié en 1606 (peu avant le départ de l'auteur pour l'Acadie), précède La défaite. Accompagné d'un sonnet en l'honneur de Pierre Dugua de Mons, vice-roi d'Henri Iv pour la Nouvelle-France, s'y greffe également une brève lettre dans laquelle le jeune avocat s'explique à sa mère ${ }^{7}$ à propos des raisons de son départ, la principale étant d'avoir été invité à participer à la fondation de la colonie par Jean de Biencourt, sieur de Poutrincourt, alors lieutenant de Dugua de Mons à Port-Royal. Se formulant telle une sorte de contrat, cette lettre fait de ce dernier la figure centrale de l'entreprise acadienne et de Lescarbot, son consignateur et promoteur officiel, à une époque où celle-ci ne bénéficiait pas d'un véritable soutien politique - Henri iv venait tout juste de révoquer le monopole de traite auquel elle devait s'adosser. Il est d'ailleurs vraisemblable que ces circonstances aient incité l'auteur à réfléchir la Nouvelle-France dans le cadre restreint de ses écrits, ne pouvant s'appuyer avec assurance sur les institutions en place ${ }^{8}$. En s'actualisant dans des ouvrages à vocation essentiellement confirmatoire en faveur d'un colonisateur qui, à la différence d'un Champlain, n'a pas écrit lui-même le récit de ses hauts faits, ce contrat correspond à un type d'engagement caractéristique des rapports entre culture et pouvoir dans la France de la fin de la Renaissance, plus précisément entre "gentilshommes de terroir et d'épée n'écriv[ant] guère» et «hommes de robe [...] jou[ant] à la fois sur le présent et [...] la postérité», "grâce à leurs rapports privilégiés avec l'intergroupe social des lettrés» ${ }^{9}$. Ce rapport entre un Lescarbot alors peu reconnu et un Jean de Biencourt détenteur de nombreux titres de noblesse, entré très jeune au service

Monseigneur le Duc de Guyse, Paris, Denis du Pré, 1575 et la Defaicte de certains Huguenotz qui tenoyent assiegé le chasteau de Crussol, faicte par M. d'Orche Couronnel [...], Lyon, Benoist Rigaud, 1577.

6. Marc Lescarbot, Adieu à la France sur l'embarquement du sieur de Poutrincourt et de son equipage faisant voile en la terre de Canadas dicte la France Occidentalle. Le vingt-sixiesme de May, 1606. Avec une lettre de l'Autheur addressante à Mademoiselle sa Mere, Rouen, Jean Petit, 1606.

7. Marc Lescarbot, «A Mademoiselle ma Mère», dans Adieu à la France, ouvr. cité, n. p.

8. Voir Brian Brazeau, «Nos ancêtres les Américains: myth and origins in early New France», Cahiers du dix-septième, vol. 12, n 1, 2008, p. 1-16.

9. Gilbert Gadroffe, La révolution culturelle dans la France des humanistes, Genève, Droz, coll. «Titre courant», 1997, p. 105. 
du duc d'Aumale, ligueur, puis défenseur d'Henri Iv après sa conversion, fera d'ailleurs l'objet d'une sorte d'attestation dans l'Adieu à la France, le poète y affirmant écrire "d'une plume apprise», pour «laiss[er] mémoire» des actions civilisatrices de son client «à la postérité $»^{10}$. Toutefois, Lescarbot semble avoir ultérieurement douté du profit qu'il tirerait d'un partage si tranché des rôles, en soutenant par exemple qu'en Nouvelle-France même, il n'aurait «pens[é] rien moins qu'à [son] histoire ${ }^{11} »$ ou — dans une tentative semblable d'accentuer son rôle de voyageur au détriment de celui d'écrivain en retranchant les vers conclusifs de La défaite des Sauvages dans sa réédition en 1609 au sein des Muses: "Cherchant dessus Neptune un repos sans repos/J'ay façonné ces vers au branle de ses flots ${ }^{12}$.»

\section{De deux dispositifs de reconnaissance: l'histoire universelle et l'épopée}

\section{La Nouvelle-France face à/dans l'histoire universelle}

Le survol du Livre III de l'édition de 1609 de l'Histoire de la Nouvelle France fait voir que les chapitres, loin de s'enchaîner au hasard, suivent l'ordre de l'histoire universelle, en ce sens qu'ils inventorient la matière selon des rubriques dont on retrouve par exemple la description dans les Deux dialogues de l'invention poétique, de la vraye cognoissance de l'histoire, de l'art oratoire, et de la fiction de la fable de Daniel d'Augé (1560), soit: la «generation », «l'education ou nourriture» des enfants, «l'institution des loix», les «ceremonies» et les «armes», mais aussi — et voilà qui apparaît plus singulier dans la mesure où Lescarbot annonce traiter des «mœurs et façons de vivre» amérindiennes —, "la manière et coustume d'exerciter les lettres ${ }^{13}$. En adoptant cette topique, le Livre in de l'Histoire ne s'intéresse donc pas tant aux peuples des Amériques en eux-mêmes qu'à certains de leurs traits et de ceux qu'on souhaite voir incarnés dans la colonie française à venir - notamment les lettres! - susceptibles de s'imbriquer dans la défense d'une Nouvelle-France dont

10. Marc Lescarbot, Adieu à la France, dans Poésies et opuscules, ouvr. cité, p. 71, v. 34-36.

11. Marc Lescarbot, Histoire de la Nouvelle France, Paris, Jean Milot, 1609, p. 663.

12. Marc Lescarbot, La défaite des Sauvages armouchiquois par le sagamos Membertou et ses alliez sauvages, dans Poésies et opuscules, ouvr. cité, p. 156, n. 488.

13. Daniel d'Augé, Deux dialogues de l'invention poétique, de la vraye cognoissance de l'histoire, de l'art oratoire, et de la fiction de la fable, Paris, Richard Breton, 1560, $f^{\circ}$ xii $r^{\circ}$. 
l'existence est pour lors essentiellement textuelle et dont la validité tient par conséquent dans l'exposition du véritable dispositif de reconnaissance que devient ici le genre de l'histoire universelle.

Le chapitre "Des Lettres» établit ainsi dès ses premières lignes la différence de l'écriture, spécialement en insistant sur le fait que les «peuples Occidentaux n'ont point l'usage des lettres». Cette différence, étudiée par Michel de Certeau à partir du récit de voyage au Brésil de Jean de Léry ${ }^{14}$, Lescarbot l'établira lui-même dans des termes fort apparentés à ceux du ministre protestant ${ }^{15}$ : non seulement les "peuples Occidentaux n'ont point l'usage des lettres", mais surtout, ils auraient «admiré de voir que par un morceau de papier [il] face [sic] conoitre [sa] volonté d'un monde à un autre, et [auraient] pens[é] qu'en ce papier il y eust de l'enchanterie» ${ }^{16}$. La défaite faisait déjà état de cette différence par la bouche même du personnage de Membertou, dont les harangues sont parsemées de références aux guerres antiques qu'il rapporterait des «anciens écrits ${ }^{17}$ ». Dans les ouvrages de Lescarbot, cette domination symbolique par les lettres — qu'il accorde ici commodément au chef des alliés des Français - s'exprime plus généralement en inscrivant dans l'ordre des mots le devenir historique d'une Nouvelle-France qui n'était encore, je le cite, qu' « un nom en l'air ${ }^{18} »$ : il fallait charger ce nom de sens, en donnant préséance à la représentation d'une expérience encore ténue du territoire et de ses habitants afin - et suivant les théories de la représentation antiques et humanistes d' «étend[re] la portée de la rationalité aux actions» relatées « et non [...] seulement à des actions qui cherchent de façon rationnelle un

14. Voir Michel de Certeau, L'écriture de l'histoire, Paris, Gallimard, 1975, p. 223 et suiv.

15. «Quant à l'escriture, soit saincte ou prophane, non seulement aussi ils ne savent que c'est, mais qui plus est, n'ayans nuls characteres pour signifier quelque chose: quand du commencement que je fus en leur pays pour apprendre leur langage, j'escrivois quelques sentences leur lisant puis apres devant, eux estimans que cela fust une sorcelerie, disoyent l'un à l'autre: N'est-ce pas merveille que cestuy-cy qui n'eust sceu dire hier un mot en nostre langue, en vertu de ce papier qu'il tient, et qui le fait ainsi parler, soit maintenant entendu de nous?» (Histoire d'un voyage faict en la terre de Bresil [1580], éd. Frank Lestringant, Paris, Librairie générale française, coll. «Le Livre de Poche/Bibliothèque classique», 1994, p. 379).

16. Marc Lescarbot, Histoire, ouvr. cité, p. 704.

17. Marc Lescarbot, La défaite, ouvr. cité, v. 124, p. 140.

18. Marc Lescarbot, Histoire, ouvr. cité, p. 208. 
but qui, lui, se trouve[rait] hors de portée de la raison ${ }^{19}$. C'est ce que manifestent par exemple, dans tout le Livre III, les sentences placées en tête des chapitres; en "encapsulant des opinions généralement acceptées ${ }^{20} »$, selon l'usage des répertoires de lieux communs, elles signalent une visée avant tout persuasive. La défaite ne faisait pas autrement, en donnant à lire une bataille entre deux tribus amérindiennes ne concernant que de très loin la jeune colonie de Port-Royal et l'expérience même des colonisateurs, confirmant par-là le rapport antagonique que pouvaient entretenir propagande impériale et mobilité, comme le souligne Paul Smethurst dans Travel Writing, Form, and Empire ${ }^{21}$.

\section{La «tentation de l'épopée ${ }^{22} »$ chez Lescarbot}

En enchâssant l'exercice de propagande dans une forme épique, l'« histoire Martiale» narrée dans La défaite place le récit colonial non seulement au cœur de l'alliance de Mars et des Muses - alliance que le chapitre «Des Lettres» rendra explicite par le biais d'un emprunt à Diodore de Sicile: «MARs Revere Les Muses ${ }^{23}{ }$ - , mais encore de Mars, des Muses et du roi «Roy tres-Chrétien, tres-Auguste et tresVictorieux Henry IIII», alliance accentuée par l'emploi des majuscules. L'Henriade de Garnier (1593), celle de Le Blanc (1604) et, avant elles, le Roy triomphant de Pontaimery (1594) avaient déjà mis en scène la figure royale en héros épique; c'est donc en empruntant cette voie générique se faisant, telles les rubriques de l'histoire universelle, dispositif de reconnaissance, que La défaite fait participer la «reconqu[ête d']Henri $\mathrm{IV}^{24}$ » à la réussite des entreprises coloniales.

19. Perrine Galland-Hallyn, «Le statut du sujet dans les théories de la représentation antiques et humanistes», dans François Cornillat et Richard Lockwood (dir.), Ethos et pathos. Le statut du sujet rhétorique, Paris, Honoré Champion, 2001, p. 33.

20. Ann Moss, Printed Commonplace-Books and the Structuring of Renaissance Thought, Oxford, Clarendon Press, 1996, p. 141.

21. Voir «Introduction», dans Paul Smethurst et Julia Kuehn (dir.), Travel Writing, Form, and Empire. The Poetics and Politics of Mobility, Oxford, Taylor et Francis, 2009, p. 2 et suiv.

22. On aura reconnu l'expression empruntée à Marie-Christine Pioffet, La tentation de l'épopée dans les Relations des jésuites, Québec, Septentrion, 1997.

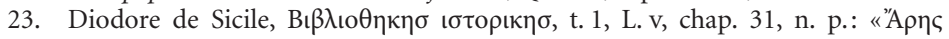

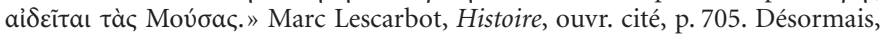
les références à cet ouvrage seront indiquées par le sigle $H N F$, suivi de la page, et placées entre parenthèses dans le corps du texte.

24. Voir Éric Thierry, La France de Henri IV, ouvr. cité, p. 256. 
En outre, les tout premiers vers du récit, qui signifieraient, selon les termes de la manchette qui les commente, que «[1']Autheur veut dire que cette histoire n'est point fabuleuse», n'en évoquent-ils pas moins deux figures épiques: «Je ne chante l'orgueil du geant Briarée/ $\mathrm{Ni}$ du fier Rodomont la fureur enivrée ${ }^{25}$.»

Toutefois, en contexte de paix retrouvée, le narrateur annonce aussitôt qu’il fera état de la guerre des autres: «Je chante Membertou [qui] joncha de morts les champs Armouchiquois/Pour la cause venger du peuple Souriquois ${ }^{26} »$, précision qui, dans les circonstances - c'est-à-dire en temps de paix retrouvée —, avait certes son importance. En effet, à l'instar du Traicté, que les lettres sont l'ornement des Roys et de l'Estat que Jean Savaron adressera en 1611 au jeune Louis XIII et qui fera participer les lettres à la montée du pouvoir absolutiste, Lescarbot brosse déjà le portrait d'un Henri iv en pacificateur favorisant les arts alors même que le royaume se voyait «traversé par les troubles et guerres continuelles ${ }^{27}$ ». De fait, l'Histoire inscrit la colonie française dans ce grand dessein, en promettant que le roi, après avoir apaisé «le tonnerre des sieges de villes et des batailles», «établira sa Nouvelle-France» tout en restaurant dans la métropole l'«ancienne splendeur [de] sa fille ainée», c'est-à-dire l'Université (HNF, p. 705-706). Il convenait par conséquent d'attribuer au vice-roi de la Nouvelle-France les qualités du monarque auquel on devait à la fois l'édit de Nantes et la Paix de Vervins (événement auquel Lescarbot fut lui-même associé ${ }^{28}$ ); La défaite crédite ainsi Pierre Dugua de Mons de la "pacifi[cation] [de] deux ou trois nations [américaines] qui de tout temps se sont fait la guerre», tout en mentionnant que l'assassinat de Panoniac fut commis «à la première occasion» (DSA, préface, p. 131-132), soit dès que le même Dugua et ses hommes eurent quitté la colonie, rendant ainsi nécessaire la présence française.

25. Marc Lescarbot, La défaite, ouvr. cité, v. 1-2, p. 135.

26. Marc Lescarbot, La défaite, ouvr. cité, v. 5-8, p. 135. Désormais, les références à cet ouvrage seront indiquées par le sigle $D S A$, suivi du vers et de la page, et placées entre parenthèses dans le corps du texte.

27. Jean Savaron, Traicté, que les lettres sont l'ornement des Roys et de l'Estat, Paris, J. de Bordeaulx, 1611, p. 17-18.

28. Voir Éric Thierry, «La Paix de Vervins et les ambitions françaises en Amérique», dans Jean-François Labourdette et coll. (dir.), Le Traité de Vervins, Paris, Presses de l'Université de Paris-Sorbonne, coll. "Centre Roland Mousnier», 2000, p. 373-390. 


\section{Coloniser à armes inégales}

Ainsi, la valeur des alliés souriquois ne tiendrait pas tant à leurs qualités propres - étant donné que même leurs ruses de guerre, pourtant mentionnées de façon quasi publicitaire dès la page de titre de la brochure, ne suffiront pas à vaincre l'ennemi -, qu'à leur propre reconnaissance de la désuétude de leurs armes. Ainsi les Souriquois n'accéderont au titre de héros que dans la mesure où, reconnaissant l'accélération de l'histoire qu'impose l'entreprise coloniale, ils constateront leur insuffisance et, pour se relever et se venger avec succès de la perte de Panoniac, ils n'auront d'autre choix que d'emprunter les armes françaises. En individualisant les Souriquois impliqués dans l'action en cours - procédé de valorisation courant chez Lescarbot ${ }^{29}$ et qui passe ici par la mention en majuscules du nom de chacun des «braves Capitaines,/Chkoudun, [...] Oagimont, Memembouré, Kichkou,/Messamoet, Ouzagat, et Anadabijou,/Medagoet, Oagimech» (DSA, v. 103-105, p. 139140 ) et «PANONiagués» (DSA, v. 107, p. 140), alors que leurs ennemis se voient désignés par un indifférencié «l'Armouchiquois»-, le récit insiste à plusieurs reprises sur les guerres continuelles des Amérindiens et sur le «feu de vengeance» (DSA, v. 132, p. 141 $1^{30}$ ) qui seul motiverait les Souriquois à engager le combat, il se verra confirmé par un autre chapitre du Livre in de l'Histoire de la Nouvelle France. Ce dernier, bien que traitant en partie des pratiques guerrières amérindiennes, ne s'y intéresse pas au premier chef. En effet, dans «De la Guerre», la sentence initiale, «De la Terre vient la Guerre», est suivie immédiatement d'une appréhension: «[Q]uand on sera établi en la Nouvelle France, quelque gourmand paraventure voudra venir enlever le travail des gens de bien et de courage» (HNF, p. 859), aussitôt tempérée par l'espoir que tous devraient placer dans cet «Etat de la France [...] maintenant trop bien affermi, grâces à Dieu, pour craindre de ces coups» (HNF, p. 859). Autrement dit: la paix retrouvée doit participer à l'effort colonial, d'autant que, apprend-on quelques lignes plus loin, les occupants du territoire

29. Comme le remarque Jack Warwick: «Lescarbot's Souriquois stand out in many anecdotes as individualized human beings, notwithstanding his dismissive attitude to them in other places» (voir «Imperial design and travel writing», dans Paul Smethurst et Julia Kuehn (dir.), Travel Writing, Form, and Empire, ouvr. cité, p. 56).

30. Voir également les v. 8, 117, 132, 186, 334 et 396. 
convoité «n'ont point leurs guerres fondées sur la possession de la terre» mais, plutôt, sur la « ressouvenance de quelque injure receue» (HNF, p. 859-860). Or, La défaite illustrait déjà ce soi-disant désintérêt du Sauvage face à la propriété territoriale, sous prétexte que, pour lui, «la terre» serait d'abord une chose à «ruiner par l'effort de la guerre» $(D S A$, v. 178, p. 143). Dans les deux textes, cette mise à l'écart du territoire à occuper correspond bien à une mise en marge d'un présent de l'histoire garant de l'existence même de la colonie ${ }^{31}$.

En effet, «De la Guerre» semble anoblir le guerrier amérindien en le comparant aux héros antiques (voir HNF, p. 859-860), la lecture parallèle de ce chapitre et de La défaite permet de nuancer une lecture trop montaignienne des écrits de Lescarbot ${ }^{32}$. En faisait du sagamos Membertou un nouvel Alexandre dont «l'heureuse victoire/ [...] lui acquit [...] une immortelle gloire» (DSA, v. 5-6, p. 135), le poème l'enferme dans un temps révolu, poussant le procédé jusqu'à le faire haranguer les siens à grand renfort de culture antique - leur donnant à entendre l'exemple d'un "Cæsar [dont] le massacre inhumain» provoqua la vengeance du "peuple Romain» (DSA, v. 119122, p. 140) — pour, au final, énoncer lui-même la désuétude de son mobile, voire son ridicule. Le procédé gagne encore en efficacité si l'on rapproche ce passage d'un autre dans lequel le narrateur compare la technique de conservation du corps de Panoniac à celle réservée aux «Rois/Que l'Ægypte pieuse embaumoit autrefois» (DSA, v. 47-48, p. 137). Encore ici, le récit semble magnifier le personnage, alors que le rite funèbre - soit les «[h] uit jours» que les proches de Panoniac passent à « [t] émoign [er] [de leur] douleur extreme» (DSA, v. 62-63, p. 137) — deviendra objet d'amusement pour les Français.

Quant à l'issue du conflit, elle viendra signera cette disqualification. En effet, si, en 1609, «De la Guerre» confinera les armes souriquoises dans un temps antérieur à l'Antiquité même — ne sont-elles pas les «premieres qui furent en usage après la creation du monde»

31. Voir Michèle Duchet, Le partage des savoirs. Discours historique et discours ethnologique, Paris, La Découverte, 1985, p. 12.

32. Telle qu'on la rencontre par exemple chez Rosalie Murphy Baum, «Early French North American Writings", dans Carla Mulford (dir.), Teaching the Literatures of Early America, New York, Modern Language Association of America, 1999, p. 117 et Réal Ouellet, "Mise en scène et fonctions de la parole amérindienne dans la relation de voyage», dans Laurier Turgeon et coll. (dir.), Transferts culturels et métissages. Amérique/Europe, $\mathrm{XVI}^{e}-\mathrm{XX}^{e}$ siècles, Paris/Québec, L'Harmattan/ Presses de l'Université Laval, 1996, p. 282. 
(HNF, p. 862)? —, en 1607, le narrateur de La défaite, au moment de raconter l'assaut final qui «venger[a] [l'] outrage» (DSA, v. 396, p. 152) dont les Souriquois furent l'objet, leur donnera le feu des armes françaises en guise de dernier recours face à "cette gent maudite aux eternelles flammes». Le chef Membertou s'occupera aussitôt à faire «tirer les Mousquets/Qu'au partir les François lui avoient tenu prets» (DSA, v. 405-406, p. 152).

Destinée à attirer l'œil, la majuscule d'accentuation à «Mousquets» confère au colonisateur, absent mais «desiré» (DSA, p. 134), un rôle central dans cette "histoire Martiale» qui promettait pourtant de relater les hauts faits des guerriers souriquois. Quant à l'emploi des armes à feu, il requalifie l'allié des Français en lui mettant entre les mains ce signe de noblesse renouvelé depuis la fin des troubles $^{33}$, ce qu'affermit la réitération immédiate du geste par un autre Souriquois, Chkoudun, qui pointera à son tour sur l'ennemi deux mousquets reçus des Français reconnaissants de l' "amour» qu'il leur a porté (voir DSA, v. 408, p. 152). Cette requalification par l'usage des armes françaises s'étend par ailleurs aux armes blanches — «fers pointus, poignars, et coutelas» (DSA, v. 251, p. 146) — placées au centre du «subtil [...] stratageme» et de la «contrefinesse» (DSA, préface, p. 132) de Membertou, qui s'en servira pour attirer les Armouchiquois avant de les retourner contre eux. Ainsi, en remportant la bataille grâce à des armes d'importation, les Souriquois justifient clairement la dévaluation de leurs propres armes face aux armes européennes «que l'esprit humain a inventé depuis deux cens ans, pour contre-carrer le tonnerre» (HNF, p. 862-863), pour reprendre les termes de «De la Guerre». Dans La défaite, les déflagrations des armes produisant en effet ce «bruit de [...] tonnerre» frappent l'Armouchiquois de stupeur (voir DSA, v. 410-412, p. 152), tout en signalant la supériorité absolue du temps nouveau qui les a vues naître. En témoignent par exemple les Nova Reperta de Jan van der Straet, recueil de gravures largement diffusé à partir des années 1580 dans lequel la poudre est à la fois célébrée pour la puissance dont elle dote l'art militaire moderne et crainte pour la dévastation qu'elle engendre - dévastation d'abord matérielle, puis morale chez des hommes «[é]branlés par la foudre et le fracas d'un tonnerre

33. Voir Russell Bouchard, Les armes à feu en Nouvelle-France, Québec, Septentrion, 1999 , p. 55 et suiv. 
provoqués de main d'homme ${ }^{34} »$. Cela étant, dans La défaite, comme dans l'A-Dieu aux François que Lescarbot aurait prononcé en 1606 à Port-Royal (imprimé en 1609 dans l'Histoire), l'usage de la poudre magnifie la prise de possession du territoire colonial:

Aymez-vous d'un Echo la babillarde voix?

Ici [c.-à-d. à Port-Royal, dans l'actuelle baie de Fundy] peut un

Echo répondre trente-fois.

Car lors que du Canon le tonnerre y bourdonne

Trente-fois à l'entour le méme coup resonne. (DSA, v. 43-46, p. 94)

\section{Acclimater le discours colonial: de l'épique au prosaïque,} de l'histoire à la fiction

À sa publication, Pierre de L'Estoille a qualifié de «bagatelle» la bataille versifiée par Lescarbot. En s'ajoutant aux autres curiosités venues d'Acadie - ce jeune Amérindien qu'on baptisa Canada et qui amusa un temps le dauphin, cet orignal qu'on laissa mourir à Saint-Germain-en-Laye —, La défaite n'aura certainement pas réussi à contrebalancer les badineries d'un Maître Guillaume qui se riait des efforts d'un Dugua de Mons «en son royaume de Canadas», «contraint luy mesmes de bescher tous les jours des espines ${ }^{35}$ » et ne comptant que sur une troupe de "milles et milles taverniers» partis «querir du safran » ${ }^{36}$. Cette rumeur, certainement plus influente que tout dispositif textuel savant, fut-il épique ou historiographique, aura peut-être incité Lescarbot à aménager dans La défaite un espace pour la facétie: que l'on parle du proverbe «[O]nques le renart ne changea sa nature,/Et de garder la foi l'homme double n'eut cure» (DSA, v. 13-14, p. 135), apprivoisant le dangereux Armouchiquois à coup de lieux communs; d'une gaillardise censée corroborer la valeur exceptionnelle d'un guerrier souriquois, "homme qui de

34. Légende originale de la gravure représentant l'invention de la poudre à canon $\left(\mathrm{n}^{\circ} 3\right.$, Pulvis pirica): "Manu quanti tonitrua atque fulmina,/Datum videtur inferis ab invidis»; nous citons la traduction de Jean-Claude Margolin, " $\mathrm{A}$ propos des Nova Reperta de Stradan », dans François Laroque et Franck Lessay (dir.), Esthétique de la nouveauté à la Renaissance, Paris, Presses de la Sorbonne Nouvelle, 2001, p. 9.

35. Maître Guillaume [Guillaume Marchand], Response de Maistre Guillaume au Soldat François. Faicte en la presence du Roy, à Fontainebleau, le huictiesme Septemb. mil six cens quatre, s. 1., s. n., 1605, p. 8.

36. Noël Mauraisin, Rencontre merveilleuse de Piedaigrette avec Maistre Guillaume revenant des Champs Elizée, s. 1., s. n., 1606, p. 3. 
six femmes/Peut, galant, appaiser les amoureuses flammes» (DSA, v. 443-444, p. 154) ou d'un Membertou soudain transfiguré en neveu de Charlemagne, d'abord solennel: «[L]e temps venu, ma trompe les appelle [i. e. ses hommes embusqués]/Lors ils viendront charger $[\ldots], /$ Et tant que durera le jour ilz frapperont, [...]. Afin qu'ici de nous long-temps on se recorde» (DSA, v. 218-222, p. 144), puis devenant prétexte à un jeu sonore: «Des trompes y avoit» parmi les cadeaux que le rusé chef présente aux Armouchiquois,

dont on ne sçavoit pas

L'usage, ni la fin du mal qu'elles couvoient.

[...]

Quand Membertou voulant étaller son credit,

Il convoque [le] peuple [de ses guerriers] embouchant une trompe,

Et trompant, les trompeurs trompeusement il trompe. (DSA, v. 252-258, p. 146)

En incorporant le Sauvage à un imaginaire vivace, ces écarts l'arrachent un instant au temps révolu où l'installe généralement le récit. Cet aspect de $L a$ défaite, et particulièrement la référence à la Chanson de Roland, fournit en outre une clé essentielle à la lecture d'un singulier passage de "De l'imposition des noms», chapitre du Livre II de l'Histoire de la Nouvelle France dans lequel Lescarbot précise qu'après la mort de Panoniac, son fils «Panoniagués quitta son nom, et fut appellé Roland par l'un des [Français]» (HNF, p. 665). Le récit de ce baptême qui n'impliqua, semble-t-il, aucun représentant religieux, se trouve appuyer subtilement un argument formulé par Lescarbot dans une épître dédicatoire au président du Parlement de Bourgogne Pierre Jeannin: en Nouvelle-France, il faudra "premièrement $[\ldots]$ ayder à établir la République, sans laquelle l'Eglise ne peut être ${ }^{37} »$. En 1610, le premier baptême collectif de la jeune colonie «gratifiera» les Souriquois des prénoms royaux, de saints ou de notables dont on recherchait évidemment la protection; dans ce contexte, le baptême de Panoniagués-Roland, qui remémore tout à la fois le plus intrépide guerrier de l'épopée française mais aussi un personnage qui hésite entre histoire et fiction, mériterait certainement un plus grand examen.

37. Marc Lescarbot, "A Monseigneur Messire Pierre Jeannin", Histoire de la Nouvelle France, Paris, Jean Milot, 1611, n. p. 